\title{
Imitative learning in Tullock contests: Does overdissipation prevail in the long run?*
}

\author{
Hiroyuki Sano ${ }^{\dagger}$ \\ Department of Economics, Otaru University of Commerce \\ 3-5-21, Midori, Otaru 047-8501, Japan
}

August 11, 2008

\begin{abstract}
This paper investigates a long-run equilibrium of the Tullock contest using an evolutionary game-theoretic approach. The finite population evolutionarily stable strategy (ESS) yields overdissipation of rent when there are increasing returns to expenditure. However, imitative behavior, considered to be a source of the evolutionary dynamics behind the ESS, is implausible because individual rationality is not always satisfied. In this paper, we attempt to specify such implicit imitative behavior and construct explicit evolutionary dynamics. Under our plausible imitation rule, we show that full dissipation may prevail in the long run as long as there are increasing returns.
\end{abstract}

JEL Classification: D72, C73

Keywords: Contests, Overdissipation, Imitative learning, Stochastically stable state

*This research was partially supported by a Grant-in-Aid for Science Research (no.17730177) from the Ministry of Education, Culture, Sports, Science and Technology of Japan.

${ }^{\dagger}$ E-mail address: sano@res.otaru-uc.ac.jp 


\section{Introduction}

The seminal work of Tullock (1980) has inspired numerous studies on rent-seeking contests. However, this has also led to the emergence of a simultaneous problem: The Nash equilibria may not exist in a rent-seeking game when there are excessive increasing returns to rent-seeking expenditure. In the static Tullock contest, the degree of marginal returns to rent-seeking expenditure is one of the most important factors for determining the rate of rent dissipation, the main area of interest in the rent-seeking theory. Tullock (1980) shows that the rate of rent dissipation is less than or equal to 1 when finite rentseekers engage in rent-seeking activities provided that a parameter $r$, which determines the degree of marginal returns, is sufficiently small. However, in the case where $r$ is excessively high, the static Tullock rent-seeking game cannot establish a pure strategy Nash equilibrium. Therefore, we are unable to confirm whether or not overdissipation of the rent occurs in the Tullock model.

If the rent-seeking game has finite players and a finite strategy space, we can verify the existence of a mixed-strategy Nash equilibrium by using a mixed extension of the strategy space. Baye, Kovenock, and de Vries (1994) confirm nonoccurrence of overdissipation of rent in the symmetric mixed-strategy Nash equilibrium of a finite rent-seeking game. Moreover, they show the existence of a symmetric mixed-strategy Nash equilibrium, which does not yield overdissipation of the rent, even in a rent-seeking game with a continuous strategy space.

Recently, Hehenkamp, Leininger, and Possajennikov (2004) adopted the concept of a finite population evolutionarily stable strategy (ESS), presented by Schaffer (1988), within the basic framework of the original Tullock contest. They revealed the existence of a finite population ESS; further, the ESS entailed overdissipation of the rent when $r$ was excessively high. However, the solution using the concept of an ESS had a drawback in that it was not possible to identify dynamic forces in order to have players adopt evolutionarily stable behaviors. Since evolutionary forces are produced through interactions among boundedly rational rent-seekers, by revealing the source of the forces, we can determine how the rationality of rent-seekers should be bounded. Therefore, it is difficult to conclude that overdissipation will occur in real-life contests if the ESS is supported by implausibly irrational behavior.

Hehenkamp et al. (2004) stated that imitative behavior among rent-seekers is obviously the source of the evolutionary force behind the finite population ESS. Therefore, in 
this paper, we attempt to specify such implicit imitative behavior and construct explicit evolutionary dynamics. Moreover, we implement discrete-time dynamics and assume that boundedly rational rent-seekers imitate one of the currently most successful strategies in the next period. The purpose of this paper is to investigate long-run equilibrium as a consequence of equilibrium selection in evolutionary dynamics and to consider whether equilibrium strategies are reasonable from the standpoint of reality.

For this purpose, we adopt the evolutionary equilibrium concept of stochastic stability instead of evolutionary stability. We demonstrate that in our imitative learning dynamics, there exists a unique stochastically stable state (SSS) and that the long-run equilibrium strategy profile constituting the unique SSS coincides with the profile of ESSs, as long as an ESS exists. Hence, the overdissipation of rent occurs in the long-run equilibrium of the SSS.

We confirm that the only factor that can cause overdissipation in the SSS is the excessively irrational behavior of rent-seekers, as they imitate the most successful strategy even though it yields a negative payoff. We consider a modified imitation rule in which such an implausibly irrational behavior is excluded and demonstrate that rent is almost always fully dissipated in the long run when there are increasing returns to rent-seeking expenditure.

The remainder of this paper is organized as follows. Section 2 reviews the static Tullock contest. Section 3 derives an SSS in imitative learning dynamics and compares the results with the finite population ESS. Section 4 defines a modified imitation rule and determines an SSS under the rule, and Section 5 discusses the results under the rule and concludes the paper.

\section{The static Tullock contest}

Consider a contest in which $N(\geq 2)$ rent-seekers compete for a prize (or rent) of size $V$. If player $i \in J=\{1,2, \ldots, N\}$ incurs an expenditure of $x_{i}$ in order to win the prize, his share of the prize is assumed to be

$$
s_{i}\left(x_{i} \mid x_{-i}\right) \equiv\left\{\begin{array}{cc}
\frac{x_{i}^{r}}{\sum_{j \in J} x_{j}^{r}} & \text { if } \max \left\{x_{j}\right\}_{j \in J}>0 \\
0 & \text { otherwise. }
\end{array}\right.
$$


where $r$ can be interpreted as a parameter that summarizes the behavior of the marginal cost of influencing the share of the prize in terms of rent-seeking expenditure, and $x_{-i}$ denotes a strategy profile with respect to all rent-seekers except for $i$. We assume that nobody can obtain any share of the prize when all rent-seekers choose zero bid. In Tullock's (1980) original model, the formulation in (1) is interpreted as the probability of winning the contest in which a winner wins the entire prize. In contrast, to avoid unnecessary complexity in the Markov process that we will explore in the subsequent sections, we assume that the rent-seeking contest is deterministic. Player $i$ attempts to maximize his payoff:

$$
\Pi_{i}\left(x_{i} \mid x_{-i}\right) \equiv s_{i}\left(x_{i} \mid x_{-i}\right) V-x_{i} .
$$

When $r \leq N /(N-1)$, in a symmetric Nash equilibrium, each of these $N$ players makes an expenditure of

$$
x^{S N E}=\frac{N-1}{N^{2}} r V .
$$

Therefore, the total rent-seeking expenditure in the symmetric Nash equilibrium amounts to

$$
X^{S N E} \equiv N x^{S N E}=\frac{N-1}{N} r V
$$

for $r \leq N /(N-1)$. However, when $r>N /(N-1)$ and finite, $x^{S N E}$ in (3) is no longer a Nash equilibrium expenditure. It is easily verifiable that the payoff to each player in (2), after the substitution of (3), will be negative. Since the strategy $x^{S N E}$ is dominated by the zero bid, the symmetric solution to the $N$ players' first-order conditions does not yield a global maximum if $r>N /(N-1)$. Thus, the rate of rent dissipation $X^{S N E} / V$ cannot be greater than one. In other words, overdissipation never occurs in the symmetric Nash equilibrium of the static Tullock contest.

\section{The dynamic Tullock contest}

Hehenkamp et al. (2004) showed that a finite population ESS does exist in the Tullock contest and that the total amount of ESS expenditure results in overdissipation of the rent if $1<r \leq N /(N-1)$. However, evolutionary stability does not specify the dynamic selection process that forces the attainment of a state comprising all the ESSs, as mentioned in Section 1. They stated that imitative behavior among rent-seekers is obviously one of the sources of the dynamics behind ESS. In this section, we explicitly 
model a situation wherein imitative behavior among rent-seekers prevails in evolutionary dynamics.

\subsection{Imitative learning dynamics}

For technical reasons, we assume that the strategy space is a finite grid, i.e., $\Gamma=$ $\{0, \Delta, 2 \Delta, \ldots, z \Delta\}$ where $\Delta \in \mathbb{R}_{++}$and $z \in \mathbb{N} ; \Delta$ can be arbitrarily small. For example, we might interpret $\Delta$ as a currency unit such as one cent. The only restriction imposed on $\Gamma$ is that $r V / N \in \Gamma$. Rent-seekers can merely observe the rent-seeking expenditures $\mathbf{x}$ $=\left(x_{1}, x_{2}, \ldots, x_{N}\right)$ and payoffs from the contest $\Pi=\left(\Pi_{1}, \Pi_{2}, \ldots, \Pi_{N}\right)$.

The dynamics proceed in discrete time, indexed by $t=0,1,2, \ldots$. The rent-seeking expenditure of player $i$ at $t$ is denoted by $x_{i}(t)$. For brevity, we occasionally denote the payoff at period $t \Pi_{i}\left(x_{i}(t) \mid x_{-i}(t)\right)$ by $\pi^{i}(t)$. There are two stages at each $t$. In the first stage, it is determined whether or not player $i$ revises his expenditure $x_{i}(t-1)$ with a common and independent probability $\delta>0$. If player $i$ decides to revise it, the decision process proceeds to the second stage in which player $i$ chooses from the set

$$
I M(t-1)=\left\{x_{j}(t-1) \in \Gamma \mid \pi^{j}(t-1)=\max \left\{\pi^{l}(t-1)\right\}_{l \in J}\right\}
$$

based on an independent probability distribution with full support. We refer to this decision rule as the "complete" imitation rule because the player completely replicates one of the most successful expenditures. We can observe from (5) that the $N$-dimensional vector of expenditures at $t$, denoted by $\mathbf{x}(t)$, is determined by an $N$-dimensional vector of expenditures at $t-1 \mathbf{x}(t-1)$. In other words, we have a discrete-time Markov process with finite state space $\Gamma^{N}{ }^{1}$ Let a monomorphic state in which all rent-seekers choose the identical expenditure $x$ be denoted by $\operatorname{mon}(x)=(x, x, \ldots, x)$. Thus, under the complete imitation rule, we obtain the following results.

Lemma 1 Any monomorphic state mon $(x)$ is in a limit set of the imitative dynamics, and any limit set is in $\{\operatorname{mon}(x)\}_{x \in \Gamma}$.

Proof. From the imitation rule, it is obvious that for any $x \in \Gamma$, the state $\operatorname{mon}(x)$ leads to a limit set of the imitative dynamics. Suppose that, contrary to our latter claim, a

\footnotetext{
${ }^{1}$ Our setting is based on the Vega-Redondo(1997) model of learning dynamics in Cournot oligopolistic competition with a discrete-time Markov process. Recently, Alós-Ferrer, Ania and Schenk-Hoppé (2000) investigated Bertrand competition in imitative dynamics using the same model .
} 
state in a limit set is a nonmonomorphic one. Since each rent-seeker can choose any $x$ $\in I M(t-1)$ with a positive probability, there is always a positive probability that the process will transit from the nonmonomorphic state to a different one. This contradicts our assumption that the nonmonomorphic state is in a limit set.

Therefore, there are $z+1$ singleton limit sets because the number of monomorphic states is equal to that of available expenditures in the finite strategy space $\Gamma$.

\subsection{The perturbed Markov process}

We now introduce an experimentation that is expected to occur with some independent probability $\epsilon>0$. Once the experimentation occurs, $x_{i}(t) \in \Gamma$ is chosen according to certain given probability distribution with full support on $\Gamma$. Thus, for each $\epsilon>0$, the perturbed Markov process has an irreducible transition matrix leading to a unique invariant distribution $\mu_{\epsilon}$ that is independent of initial conditions and assigns positive probability to all states in $\Gamma^{N}$. However, the invariant distribution $\mu_{\epsilon}$ depends on the occurrence of experimentation $\epsilon$; moreover, the invariant distribution is expected to concentrate almost all of its probability on a few states as $\epsilon \rightarrow 0$ (see Foster and Young [1990]; Kandori, Mailath, and Rob [1993]; and Young [1993]). In other words, we can investigate the relative robustness among the monomorphic states as the occurrence of experimentation vanishes. Hence, we focus on the limit invariant distribution of the perturbed Markov process as $\epsilon \rightarrow 0$, denoted by $\mu^{*} \equiv \lim _{\epsilon \rightarrow 0} \mu_{\epsilon}$. If a state is assigned some positive probability according to the limit invariant distribution $\mu^{*}$, then the state is considered to be stochastically stable. A stochastically stable set comprises all the states with positive probabilities. Stochastically stable states are, intuitively, the states that are most likely to be observed over the long run when experimentation is a rare occurrence.

Recent evolutionary literature reveals the use of techniques provided by Freidlin and Wentzell (1984) to find an SSS. Any ordered pair of states is referred to as an "arrow," which is denoted by $\left(\mathbf{x}^{\prime}, \mathbf{x}^{\prime \prime}\right)$ for $\mathbf{x}^{\prime}, \mathbf{x}^{\prime \prime} \in \Gamma^{N}$. For each $\mathbf{x} \in \Gamma^{N}$, an $\mathbf{x}$-tree is a collection of the arrows such that every $\mathbf{x}^{\prime} \in \Gamma^{N} \backslash\{\mathbf{x}\}$ is the first element of the arrow, and for every $\mathbf{x}^{\prime} \in \Gamma^{N} \backslash\{\mathbf{x}\}$, there is a path $\left\{\left(\mathbf{x}^{0}, \mathbf{x}^{1}\right),\left(\mathbf{x}^{1}, \mathbf{x}^{2}\right), \ldots,\left(\mathbf{x}^{s-1}, \mathbf{x}^{s}\right)\right\}$ where $\mathbf{x}^{0}=\mathbf{x}^{\prime}$ and $\mathbf{x}^{s}=\mathbf{x}$. The cost of the arrow $\left(\mathbf{x}^{\prime}, \mathbf{x}^{\prime \prime}\right)$ is the minimal number of experimentations required for the transition from $\mathbf{x}^{\prime}$ to $\mathrm{x}^{\prime \prime}$ to occur with a positive probability. The cost of a $\mathbf{x}$-tree is the sum of the costs of all the arrows that belong to the $\mathbf{x}$-tree. The lowest cost among all $\mathbf{x}$-trees is the stochastic potential of $\mathbf{x}$. According to Young (1993), the limit 
invariant distribution $\mu^{*}$ only assigns positive probability to the states having a minimum stochastic potential. In other words, of all trees in every limit state in an unperturbed Markov process, the state with a minimal cost tree is stochastically stable. Further, a stochastically stable set comprises all the limit states with minimal cost trees.

\subsubsection{SSS in the complete imitation rule}

Suppose that $m(\leq N-1)$ rent-seekers choose $x$ and $N-m$ rent-seekers choose $\hat{x}$. We occasionally refer to the former as "mutants" and the latter as "incumbents" according to the convention of literature on the evolutionary game theory. Defining $\hat{x}_{-i}(m) \equiv$ $(\underbrace{x, \ldots x}_{m}, \underbrace{\hat{x}, \ldots, \hat{x}}_{N-1-m})$ and $\hat{x}_{-i}(m-1) \equiv(\underbrace{x, \ldots x}_{m-1}, \underbrace{\hat{x}, \ldots, \hat{x}}_{N-m})$, the payoff for a player choosing $x$ is

$$
\Pi_{i}\left(x \mid \hat{x}_{-i}(m-1)\right)=\frac{x^{r}}{m x^{r}+(N-m) \hat{x}^{r}} V-x .
$$

The payoff for a player choosing $\hat{x}$ is

$$
\Pi_{i}\left(\hat{x} \mid \hat{x}_{-i}(m)\right)=\frac{\hat{x}^{r}}{m x^{r}+(N-m) \hat{x}^{r}} V-\hat{x}
$$

Subtracting (7) from (6), we obtain the following relative payoff:

$$
\Phi(x, \hat{x} ; m)=\frac{x^{r}-\hat{x}^{r}}{m x^{r}+(N-m) \hat{x}^{r}} V-x+\hat{x} .
$$

The relative payoff is useful in computing the cost of an arrow. If $\Phi(x, \hat{x} ; N-1) \leq 0$, then the minimal number of experimentations required for the transition from mon $(x)$ to mon $(\hat{x})$ to occur with a positive probability, i.e., the cost of the arrow, is just one. Therefore, if $\hat{x}$ is a global maximizer of $\Phi(x, \hat{x} ; N-1)$, then $\Phi(x, \hat{x} ; N-1) \leq 0$ for any $x \neq \hat{x}$. Thus, the stochastic potential of mon $(\hat{x})$ is $z$, since for every $x \neq \hat{x}$, the cost of the arrow from mon $(x)$ to mon $(\hat{x})$ is one. Thus, mon $(\hat{x})$ has the minimum stochastic potential since $z$ is the number of monomorphic states except for mon $(\hat{x})$.

Lemma 2 If $r \leq N /(N-m)$ for given $m \in[1, N-1]$, then $x=r v$ is a global maximizer of $\Phi(x, r v ; m)$ where $v \equiv V / N$.

Proof. At present, suppose that player $i$ chooses $x_{i} \in C$ where $C$ is a continuum of expenditures on the interval $[0, \infty)$ in $\mathbb{R}_{+}$. The first-order condition for maximizing 
$\Phi(x, \hat{x} ; m)$ in $(8)$ with respect to $x$ is

$$
\frac{\partial}{\partial x} \Phi(x, \hat{x} ; m)=\frac{N x^{r-1} \hat{x}^{r}}{\left[m x^{r}+(N-m) \hat{x}^{r}\right]^{2}} r V-1=0 .
$$

When $x=\hat{x}=r v$,

$$
\frac{\partial}{\partial x} \Phi(r v, r v ; m)=\frac{1}{N r v} r V-1=0 .
$$

Thus, we obtain $x=r v$. The second-order derivative of $\Phi(x, r v ; m)$ with respect to $x$ is

$$
\frac{\partial^{2}}{\partial x^{2}} \Phi(x, r v ; m)=\frac{(r-1)(N-m)(r v)^{r}-(r+1) m x^{r}}{\left[m x^{r}+(N-m)(r v)^{r}\right]^{3}} x^{r-2}(r v)^{r} N r V .
$$

Therefore, the sufficient condition for $r v$ locally maximizing $\Phi(x, r v ; m)$ is

$$
\lambda(m) \equiv \frac{(r-1)(N-m)}{(r+1) m}<1 .
$$

It is evident from (11) that the function $\Phi(x, r v ; m)$ is strictly concave (respectively, convex) if $x>$ (respectively, $<) \lambda^{\frac{1}{r}}(m) r v$. For $r \leq 1, \Phi(x, r v ; m)$ is concave at $x=0$ and strictly concave at any $x>0$ since $\lambda(m) \leq 0$. Hence, $x=r v$ is a global maximizer of $\Phi(x, r v ; m)$ for any $r \leq 1$.

Next, consider the case in which $r>1$. When $r>1, \lambda(m)$ is positive in that $\Phi(x, r v ; m)$ is strictly convex for any $x<\lambda^{\frac{1}{r}}(m) r v$ and strictly concave for any $x>$ $\lambda^{\frac{1}{r}}(m) r v$. Thus, $\lambda^{\frac{1}{r}}(m) r v$ is the only inflection point of $\Phi(x, r v ; m)$. This implies that either 0 or $r v$ can globally maximize $\Phi(x, r v ; m)$ if the inequality of (12) is satisfied, i.e., $\lambda(m)<1$. When $m \geq N / 2$, (12) holds regardless of $r$. Rearranging (12) yields $r$ $<N /(N-2 m)$ if $m<N / 2$. In other words, (12) can be satisfied for $r<N /(N-2 m)$ when $m<N / 2$. Substituting 0 and $r v$ for $x$ and $\hat{x}$, respectively, in (8), we obtain

$$
\Phi(0, r v ; m)=\frac{r(N-m)-N}{N(N-m)} V
$$

For any $r \leq N /(N-m)$, we have $\Phi(0, r v ; m) \leq 0$. Since $\lambda(m)<1$ for any $r<$ $N /(N-2 m)$ and $\Phi(r v, r v ; m)=0, r v$ is a global maximizer of $\Phi(x, r v ; m)$ for any $r$ $\leq N /(N-m)$. Figure 1 depicts a configuration of the graph of $\Phi(x, r v ; m)$ in the case that $1<r<N /(N-m)$. From the assumption that $r v$ is contained in the finite grid $\Gamma, r v$ can also be a global maximizer of $\Phi(x, r v ; m)$ when a strategy space is $\Gamma$. 


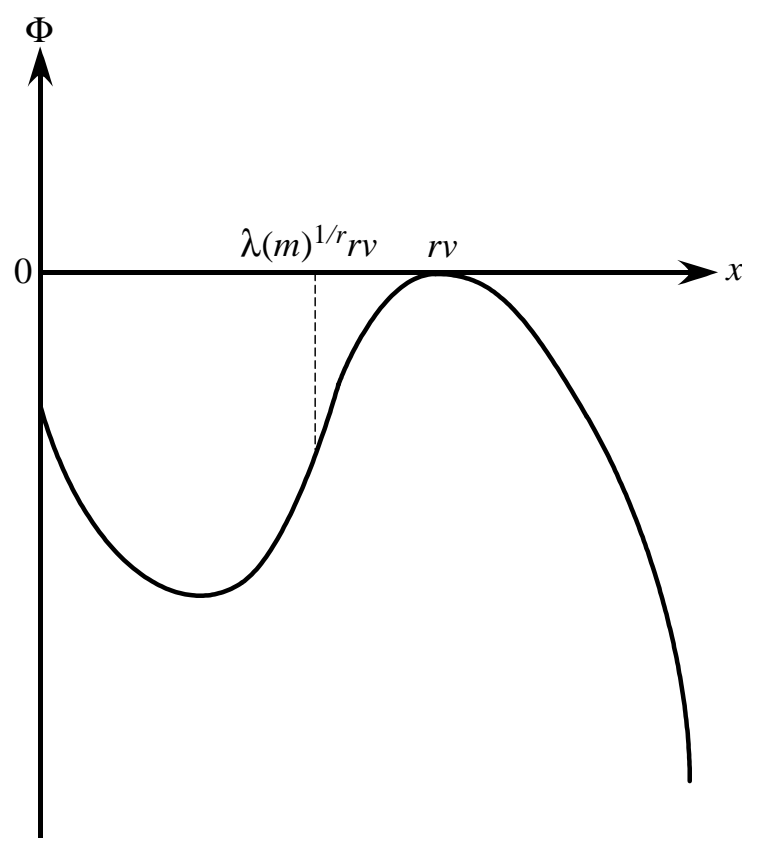

Figure 1: Configuration of the graph of the function $\Phi(x, r v ; m)$

Lemma 2 states that a player choosing $r v$ acquires the highest payoff when the number of other players choosing $x \neq r v$, denoted by $m$, is such that $r \leq N /(N-m)$.

Proposition 1 For every $r \leq N$, mon $(r v)$ is an SSS in the imitative learning dynamics of the Tullock contest under the complete imitation rule. Moreover, for every $r<$ $N /(N-1)$, mon (rv) is a unique SSS, while for any $r \in[N /(N-1), N],\{\operatorname{mon}(x)\}_{x \in[0, V]}$ is a stochastically stable set.

Proof. Lemma 1 states that no nonmonomorphic state can be included in the stochastically stable set. When $m=N-1$, from Lemma 2, $r v$ is a global maximizer of $\Phi(x, r v ; N-1)$ for any given $r \leq N$. Thus, we have

$$
\Phi(x, r v ; N-1) \leq 0 \text { for any } x \in \Gamma \backslash\{r v\},
$$

if $r \leq N$. The cost of mon ( $r v)$-tree is $z$ since the inequality (14) implies that for every $x \in \Gamma \backslash\{r v\}$, the cost of the arrow from mon $(x)$ to mon $(r v)$ is just one. Therefore, the stochastic potential of mon $(r v)$ is equal to $z$. Clearly, $z$ is the minimum stochastic potential. Hence, mon ( $r v)$ is contained in the stochastically stable set. 
For uniqueness in the case where $r<N /(N-1)$, we must verify that no tree rooted in mon $(x)$ except for mon ( $r v$ ) has the minimum stochastic potential $z$. Lemma 2 states that $r v$ is a global maximizer of $\Phi(x, r v ; 1)$ for any given $r<N /(N-1)$, or equivalently

$$
\Phi(x, r v ; 1)<0 \text { for any } x \in \Gamma \backslash\{r v\}
$$

if $r<N /(N-1)$. Eq. (15) implies that for any $x \in \Gamma \backslash\{r v\}$, the cost of the arrow from mon ( $r v)$ to mon $(x)$ is more than one. In other words, the transition from mon ( $r v$ ) to any other monomorphic state involves more than one experimentation. Therefore, the stochastic potential of the tree rooted in mon $(x)$ for any $x \in \Gamma \backslash\{r v\}$ is more than $z$.

Next, consider the case in which $N /(N-1) \leq r<N$. In this case, we have $\Phi(0, r v ; 1)$ $\geq 0$, which implies that the transition from mon $(r v)$ to mon (0) can occur with one experimentation. Moreover, from (14), for every $x \in \Gamma \backslash\{0, r v\}$, the cost of the arrow from mon $(x)$ to mon $(r v)$ is one. The sum of the costs of these arrows is $z-1$. Thus, there is a tree rooted in mon (0) which has the minimum stochastic potential $z$. Next, let us consider a tree rooted in mon $\left(x^{\prime}\right)$ where $x^{\prime}$ is an expenditure in $(0, V] \cap \Gamma \backslash\{r v\}$. We can easily verify that the transition from mon $(0)$ to mon $(x)$ for any $x \in(0, V] \cap \Gamma$ can be achieved with one experimentation since $\Phi(x, 0 ; 1) \geq 0$. Therefore, the cost of the arrow from mon (0) to mon $\left(x^{\prime}\right)$ is one. In addition to this, the cost of the arrow from mon (rv) to mon (0) is one, and the sum of the costs of arrows from mon (x) to mon (rv) for all $x \in \Gamma \backslash\left\{0, r v, x^{\prime}\right\}$ is $z-2$. Hence, the cost of a tree rooted in mon $\left(x^{\prime}\right)$ for any $x^{\prime}$ $\in(0, V] \cap \Gamma \backslash\{r v\}$ can be $z$.

For any positive $r \leq N$, the SSS mon (rv) leads to the aggregate rent-seeking expenditure of $N r v=r V$. Thus, the rate of rent dissipation in the SSS is equal to $r$. Therefore, when $r$ is greater than one but not greater than $N$, overdissipation occurs frequently in the long run where the SSS can be overwhelmingly obtained.

According to Hehenkamp et al. (2004), there exists a unique ESS for any positive $r$ $\leq N /(N-1)$. The ESS derived is identical to the individual strategy $r v$ constituting the SSS derived above. However, the set of $r$ ensuring the existence of the ESS is included in the set of $r$ for our SSS, with the exception of the case in which $N=2$. In other words, when $r$ is given as a value such that $N /(N-1)<r \leq N, r v$ is the strategy constituting one of the SSSs but not an ESS. This difference increases as the number of rent-seekers increases. In other words, the larger the $N$, the larger is the upper limit of $r$ for the SSS 
and the smaller is the upper limit for the ESS. Moreover, as $N \rightarrow \infty$, the upper limit for the ESS converges to 1; thus, the possibility of overdissipation in the ESS vanishes. In contrast, in our imitative dynamics, the infinitely large number of rent-seekers may result in a higher rate of overdissipation since it allows the existence of the SSS $r v$ for any large $r$.

However, the ESS in Hehenkamp et al. (2004) is a finite population ESS that is defined in Schaffer (1988), and therefore, $N$ is assumed to be finite such that there is a range of $r$ that induces overdissipation. It is necessary to satisfy the following condition so that $r v$ is a finite population ESS in the Tullock contest.

$$
\Phi(x, r v ; 1)<0 \text { for any } x \neq r v \text {. }
$$

This implies that if a single rent-seeker chooses any strategy $x \neq r v$ and other $N-1$ rent-seekers choose $r v$, then the payoff for the rent-seeker choosing $x \neq r v$ must be lower than that for the other one. Since the only candidates for a global maximum of $\Phi(x, r v ; 1)$ are 0 and $r v$, the ESS requires that a single rent-seeker choosing the zero bid obtains a strictly lower payoff than the other $N-1$ rent-seekers who choose $r v$. In other words, each of the $N-1$ rent-seekers choosing $r v$ must obtain a positive payoff, i.e., $N v /(N-1)-r v>0$, which yields $r<N /(N-1)$. In order for $r v$ to constitute an SSS given the zero bids for $N-1$ rent-seekers, a single rent-seeker choosing $r v$ has to merely obtain a nonnegative payoff, while the other $N-1$ rent-seekers retain zero payoffs, as denoted in (13). Therefore, it is required that $N v-r v \geq 0$ or equivalently $r \leq N$.

According to Alós-Ferrer and Ania (2005), in a rent-seeking contest, a profile consisting of $N$ identical ESSs, which are globally stable, coincides with the unique SSS of the imitative dynamics with experimentation if the globally stable ESS exists. The global stability of an ESS requires that a player choosing the ESS has a higher payoff than each of $m$ players choosing any other strategy for every $m \in[1, N-1]$. It is evident from Lemma 2 that for any $m \in[1, N-1]$ and $x \neq r v, \Phi(x, r v ; m)<0$ if $r<N /(N-1)$, which implies that $r v$ is a globally stable ESS for any $r<N /(N-1)$. That is, the necessary condition for $r v$ to be a globally stable ESS is $r<N /(N-1)$. This inequality is also a necessary condition for mon ( $r v)$ to be a unique SSS. Our result serves as an example indicating that the strategy constituting one of the SSSs is not a globally stable ESS when a stochastically stable set is not singleton, since for any $r \in[N /(N-1), N]$, mon ( $r v)$ is one of the SSSs, but $r v$ is not an ESS. 
With respect to any given $r \geq N /(N-1)$, it is difficult to predict the states that will be mostly observed in the long run and whether or not overdissipation will occur frequently. This is because mon $(x)$ for any $x \in[0, V]$ can be an SSS. As is evident, the result would include an anomaly. For an arbitrary small $\eta$, we obtain the only SSS mon $(r v)$ for given $r=N /(N-1)-\eta$ while many SSSs can be attained for given $r$ $=N /(N-1)$. This indicates that there would be room for reconsidering the complete imitation rule defined in (5).

\subsubsection{SSS in the cautious imitation rule}

We examine an SSS in an alternative imitation rule where each player gradually brings his expenditure close to the most successful strategy instead of completely and immediately imitating the strategy. For example, in the complete imitation rule considered above, the transition from mon ( $r v$ ) to mon (0) can occur with one experimentation. The reason behind only one experimentation being able to yield the transition is that all the $N-1$ incumbent players imitate zero expenditure, which is the most successful strategy with positive probability since $\Phi(0, r v ; 1)>0$ for $r \geq N /(N-1)$. However, while an incumbent obtains zero payoff if he immediately imitates this expenditure, he may be able to acquire a positive payoff if he continues choosing $r v$ when many other incumbents choose to change their expenditures to zero. Every player may experience such a case in the past periods if $\delta<1$. Therefore, the players are able to recognize that complete and immediate imitation involves an opportunity cost. On the other hand, if few incumbents imitate the zero bid, they would be aware of the opportunity cost involved in choosing $r v$. Even in the case that all players revise their strategy, i.e., $\delta=1$, each player might regard such an opportunity cost to be more or less significant unless the players have common knowledge regarding the behavioral rule.

The possible existence or perception of opportunity costs in changing and retaining strategies would make rent-seekers exercise caution. In light of this consideration, we alternatively consider a "cautious" imitation rule, wherein each player's decision process in each period consists of three stages. In the first stage of period $t$, each player revises his expenditure of $x_{i}(t-1)$ with probability one. In the second stage, player $i$ identifies a target from $I M(t-1)$ in (5) based on an independent probability distribution with full support. Finally, in the third stage, player $i$ chooses his expenditure of $x_{i}(t-1)+\Delta$ (respectively, $x_{i}(t-1)-\Delta$ ) if the target chosen is more (respectively, less) than $x_{i}(t-1)$, 
and his strategy at $t-1$ is maintained if the target is equal to that strategy. That is, each player gradually makes an expenditure close to one of the currently most successful strategies provided that the strategy continues to be the most successful in the subsequent periods.

Proposition 2 For every $r<N$, mon $(r v)$ is an SSS in the learning dynamics of the Tullock contest under the cautious imitation rule. Moreover, there exists a unique stochastically stable set that is given by:

(i) $\{$ mon $(r v)\}$ for any positive $r<N /(N-1)$

(ii) $\{\operatorname{mon}(x)\}_{x \in\left[\frac{N}{N-1} v-\Delta, r v\right] \cap \Gamma}$ for any $r \in[N /(N-1), N /(N-2)]$.

Proof. See Appendix.

In the case where $r<N /(N-1)$, mon ( $r v)$ is the only SSS; additionally, we have only two SSSs, mon $(r v-\Delta)$ and mon $(r v)$, if $r$ is equal to $N /(N-1)$. Further increase in $r$ increases the number of SSSs because the stochastically stable set is $\left[\frac{N}{N-1} v-\Delta, r v\right] \cap \Gamma$. In other words, the higher the value of $r$, the less precise is the prediction that a long-run equilibrium will survive in the evolutionary process. However, at $\operatorname{mon}\left(\frac{N}{N-1} v-\Delta\right)$, the total amount of rent-seeking expenditure is $\frac{N}{N-1} V-N \Delta$ which is greater than $V$ if $\Delta<$ $\frac{1}{N-1} v$. Therefore, overdissipation occurs at every monomorphic state in $\left[\frac{N}{N-1} v-\Delta, r v\right] \cap$ $\Gamma$ for a sufficiently small $\Delta$.

\section{The modified imitative learning rule}

In both the imitation rules described in Section 3.1, even though the absolute payoff for the most successful rent-seeker is negative, the other rent-seekers are likely to imitate or target the choice of the most successful rent-seeker with a positive probability in the next period. However, this imitation rule does not appear to be plausible. Even rentseekers without complete information and computation ability would know a priori that if they choose zero expenditure, their absolute payoff could be zero regardless of the other players' strategies. Therefore, they may infer that the most successful strategy should not be one of the observable positive expenditures but zero expenditure. Considering this, it is worth investigating the following modified cautious imitation rule: Player $i$, at the 
second stage in period $t$, finds a target from the set

$$
M I M(t-1)=\left\{x_{j}(t-1) \in \Gamma \mid \pi^{j}(t-1)=\max \left\{\pi^{l}(t-1)\right\}_{l \in J} \& \pi^{j}(t-1) \geq 0\right\},
$$

based on an independent probability distribution with full support if $M I M(t-1) \neq$ $\varnothing$; otherwise, he targets $x_{i}=0$. At the third stage in period $t$, player $i$ brings his expenditure close to the target by $\Delta$. In other words, the rent-seekers are expected to target an expenditure based on observations only when they find one that results in a positive payoff. If not, they are likely to decrease their expenditures by $\Delta$ targeting zero expenditure. We can refer to $\pi^{j}(t-1) \geq 0$ appearing in (17) as "individual rationality" or "participation" constraint because the reservation payoff for each rent-seeker equals zero. $^{2}$ Additionally, we assume that $v$ is included in the finite grid $\Gamma$.

Proposition 3 Under the modified cautious imitation rule, there exists a unique SSS in the learning dynamics of the Tullock contest, which is given by:

(i) mon ( $r v$ ) for any positive $r \leq 1$

(ii) mon ( $v$ ) for any finite $r>1$.

Proof. See Appendix.

A monomorphic state mon $(x)$ for any $x>v$ is excluded from the limit sets of the unperturbed Markov process owing to the modified imitation rule since any strategy $x$ $>v$ conflicts with the individual rationality for each rent-seeker. Violation of individual rationality entails overdissipation in the contest. Therefore, under the modified imitation rule, which is compatible with individual rationality, overdissipation cannot prevail in the long run. In the SSS mon $(v)$ for $r \geq 1$, each rent-seeker spends $v$ in order to obtain a share of the rent. Consequently, the sum of the expenditures in the per-period contest amounts to $V$; thus, the rent $V$ is fully dissipated for any finite $r \geq 1$ in the unique SSS.

Remark 1 Note that mon $(v)$ is not stochastically unstable even though a transition from mon $(v)$ to some state occurs with only one experimentation. For instance, consider the case in which a player chooses $v+\Delta$ by way of experimentation in the state of mon $(v)$. In this case, we obtain $\Phi(v+\Delta, v ; 1)>0$ by considering $\Delta$ as a sufficiently small value. This implies that the transition from mon $(v)$ to mon $(v+\Delta)$ can be realized

\footnotetext{
${ }^{2}$ The term "individual rationality" implies that potential players participate in a game only when they anticipate their payoffs from the game to be more than or equal to their reservation payoff.
} 
with one experimentation. However, mon $(v+\Delta)$ is transient and thus can return to mon $(v)$ without experimentation owing to the modified cautious imitation rule. In short, we should not judge a state as being unstable because it can move to other states with one experimentation. In this respect, stochastic stability is different from the concept of a finite population ESS.

Remark 2 Hehenkamp et al. (2004) demonstrated that the finite population ESS coincides with a symmetric Nash equilibrium strategy when each player maximizes his relative payoff by assuming the strategies of all the other players as given. Since the relative payoff becomes zero at the ESS, we may interpret that individual rationality is satisfied with respect to the relative payoff and therefore is not violated in the evolutionary equilibrium. However, the objective of maximizing a relative payoff is the consequence of an economic natural selection in which the dynamic forces are driven by some imitation rule. Our concern regarding individual rationality does not lie in the consequence but in the source of the economic natural selection. Since the concept of a finite population ESS does not express a dynamic process, our criticism regarding individual rationality could not directly apply to the result in the finite population ESS of the Tullock contest. Nonetheless, if the objective of relative payoff maximization is the consequence of imitative learning dynamics conflicting with individual rationality, we suspect that the consequence may not be valid because the premises of the model are implausible.

\section{Conclusions}

We have modeled imitative behavior explicitly in the dynamic Tullock contest and have demonstrated that the unique SSS is consistent with the finite population ESS derived in Hehenkamp et al. (2004). Moreover, an SSS exists in the contest with a higher $r$ such that the finite population ESS does not exist, although the SSS is no longer unique.

Complete imitative behavior among rent-seekers would be rather problematic even though we agree with the assumption that the rationality of rent-seekers is bounded. Under the complete imitation rule, each rent-seeker is so indifferent to other rent-seekers' choices that he is not able to recognize the fact that a greater change in his strategy may involve some opportunity costs. Therefore, we have presented the cautious imitation rule under which each incumbent gradually brings his expenditure close to the most successful strategy. The stochastically stable set under the cautious imitation rule is smaller than 
that under the complete imitation rule so that we can be more precise in predicting the result that will be obtained in the long run.

One source of overdissipation is the lack of individual rationality. In other words, rent-seekers participate in the contest without individual rationality even though they incur a negative payoff, and thus, the sum of individual expenditures is more than the value of the rent. However, the notion of the ESS cannot exclude any imitative behavior that results in a negative individual payoff, although it is difficult to find such irrational behavior in any real-life contest. The finite population ESS derived by Hehenkamp et al. (2004) does not satisfy the individual rationality for any $r$ in the interval of $(1, N /(N-1)$ ] such that it yields overdissipation. Therefore, in order for the ESS to be compatible with individual rationality, our analyses must be restricted to the case where $r \leq 1$. However, by reconciling individual rationality with the imitation of the most successful behavior, we have shown that there exists an SSS for any finite $r$.

Corcoran (1984) and Corcoran and Karels (1985) have studied a long-run equilibrium of the rent-seeking contest endogenizing the entry decisions of rent-seekers. They showed that the number of rent-seekers participating in the contest increases or decreases until the payoffs for active rent-seekers fall to zero, to the extent that $1<r \leq N /(N-1)$. Therefore, in the long-run equilibrium of their free entry model, for any $r \in(1, N /(N-1)]$, individual rationality or participation constraints are satisfied, and thus, the rent is fully dissipated. In this paper, we have shown that for any finite $r \geq 1$, the rent is fully dissipated in the only SSS under the modified cautious imitation rule. This implies that overdissipation is a rare occurrence in the long run and that the "full-dissipation hypothesis" may be valid even though $r$ is greater than 1 .

\section{Appendix}

Proof of Proposition 2. Suppose that at $t$, only one player chooses $r v$ while all the other $N-1$ players choose any $x \in \Gamma \backslash\{r v\}$. Then, all $N-1$ players who have chosen $x$ at $t$ change their strategy to $x+\Delta$ (respectively, $x-\Delta$ ) at $t+1$ if $x<$ (respectively, $>$ ) $r v$ from (14) according to the cautious imitation rule. The inequality (14) and cautious imitation rule imply that the process of such transition without experimentation continues until mon $(r v)$ is attained. That is, for every $x \in \Gamma \backslash\{r v\}$, the transition from mon $(x)$ to mon ( $r v$ ) occurs with one experimentation. Hence, for any positive $r \leq N$, mon $(r v)$ is an 
SSS since the stochastic potential of mon ( $r v$ ) is equal to $z$. To verify (i), we can use the result from Lemma 2 that $\Phi(x, r v ; 1)<0$ for any $x \in \Gamma \backslash\{r v\}$ if $r<N /(N-1)$. This result implies that the transition from mon $(r v)$ to mon $(x)$ for any $x \in \Gamma \backslash\{r v\}$ entails more than one experimentation. Hence, the stochastic potential of mon $(x)$ for every $x$ $\in \Gamma \backslash\{r v\}$ cannot be the minimum.

Next, to prove (ii), we investigate the case where $N /(N-1) \leq r \leq N /(N-2)$.

Lemma 3 For any given finite $r \geq \frac{N}{N-1}$ and for every $\hat{x} \in\left[\frac{N}{N-1} v, r v\right]$, there exists a nonnegative $\bar{x} \in C$ such that $\Phi(x, \hat{x} ; 1)>0$ for any $x<\bar{x}, \Phi(x, \hat{x} ; 1)<0$ for any $x$ $\in(\bar{x}, \hat{x})$ and $\Phi(\bar{x}, \hat{x} ; 1)=0$.

Proof. From (8) and (9), we have $\Phi(0, \hat{x} ; 1)=-\frac{N}{N-1} v+\hat{x} \geq 0$ and $\frac{\partial}{\partial x} \Phi(0, \hat{x} ; 1)=-1$, respectively. Moreover, $\Phi(x, \hat{x} ; 1)$ is strictly convex for $x<\lambda^{\frac{1}{r}}(1) \hat{x}$ and strictly concave for $x>\lambda^{\frac{1}{r}}(1) \hat{x}$. Therefore, $\Phi(x, \hat{x} ; 1)=0$, at most, has three real roots, one of which is clearly $\hat{x}$. Since $\hat{x} \leq r v$, we obtain $\frac{\partial}{\partial x} \Phi(\hat{x}, \hat{x} ; 1)=\frac{r v}{\hat{x}}-1 \geq 0$ from (9). Thus, there is just one real root $\bar{x}$ in the interval $[0, \hat{x})$ at which $\frac{\partial}{\partial x} \Phi(\bar{x}, \hat{x} ; 1)<0$. This completes the proof.

Lemma 3 states that $\Phi(x, r v ; 1) \geq 0$ for any $x \leq \bar{x}$. (See Figure 2.) This implies that each of the $N-1$ incumbents changes from $r v$ into $r v-\Delta$ at $t+1$ according to the cautious imitation rule once only one mutant chooses any $x \leq \bar{x}$ at $t$. Moreover, since for every $\hat{x} \in\left[\frac{N}{N-1} v, r v\right]$, the equation $\Phi(x, \hat{x} ; 1)=0$ has a unique solution $x=\bar{x}$ $\in[0, \hat{x})$, solving the equation $\Phi(\bar{x}, \hat{x} ; 1)=0$ for $\bar{x}$ yields an explicit function $\bar{x}=\chi(\hat{x})$ where $\chi:\left[\frac{N}{N-1} v, r v\right] \longrightarrow[0, \hat{x})$.

Lemma 4 For given any finite $r \geq \frac{N}{N-1}, \bar{x}$ increases with $\hat{x} \in\left[\frac{N}{N-1} v, r v\right)$, and $\chi\left(\frac{N}{N-1} v\right)$ $=0$.

Proof. From the proof of Lemma 3, $\bar{x} \in(0, \hat{x})$ is a real root of $\Phi(x, \hat{x} ; 1)=0$ and $\frac{\partial}{\partial x} \Phi(\bar{x}, \hat{x} ; 1)<0$. Letting $\varphi \equiv \Phi(\bar{x}, \hat{x} ; 1)$ and differentiating $\varphi$ with respect to $\bar{x}$ yield

$$
\frac{\partial \varphi}{\partial \bar{x}}=\frac{\bar{x}^{r-1} \hat{x}^{r}}{\left[\bar{x}^{r}+(N-1) \hat{x}^{r}\right]^{2}} N r V-1<0 .
$$

Similarly, differentiating $\varphi$ with respect to $\hat{x}$, we obtain

$$
\frac{\partial \varphi}{\partial \hat{x}}=-\frac{\bar{x}^{r} \hat{x}^{r-1}}{\left[\bar{x}^{r}+(N-1) \hat{x}^{r}\right]^{2}} N r V+1>0,
$$




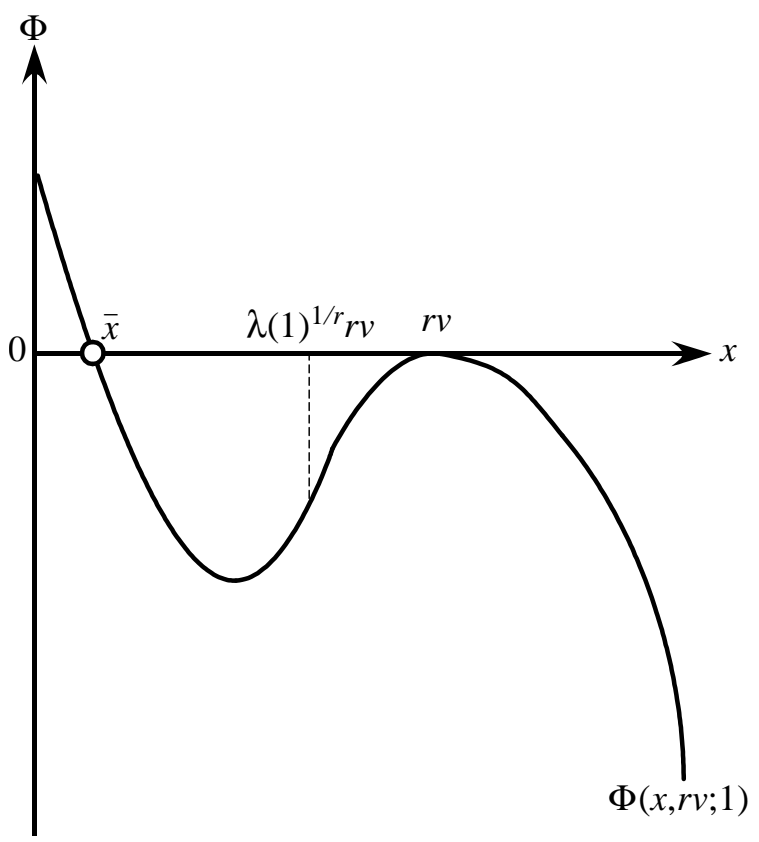

Figure 2: Configuration of the graph of the function $\Phi(0, \hat{x} ; 1)$ for $r>\frac{N}{N-1}$

since $\bar{x}<\hat{x}$ and $\frac{\partial \varphi}{\partial \bar{x}}<0$ from (A1). Therefore, differentiating $\Phi(\chi(\hat{x}), \hat{x} ; 1)=0$ with respect to $\hat{x}$, we obtain

$$
\chi^{\prime}(\hat{x}) \equiv \frac{d \bar{x}}{d \hat{x}}=-\frac{\partial \varphi / \partial \hat{x}}{\partial \varphi / \partial \bar{x}}>0 .
$$

From (8) and (9), we have $\Phi\left(0, \frac{N}{N-1} v ; 1\right)=-\frac{N}{N-1} v+\frac{N}{N-1} v=0$ and $\frac{\partial}{\partial \bar{x}} \Phi\left(0, \frac{N}{N-1} v ; 1\right)$ $=-1$, which implies that $\bar{x}=0$ when $\hat{x}=\frac{N}{N-1} v$.

Suppose that at $t$, a rent-seeker chooses a nonnegative $x^{\prime} \leq \bar{x}$ while the other $N-1$ rent-seekers choose $r v$. Then, each of the $N-1$ incumbents changes into $r v-\Delta$ at $t+1$, as mentioned above, and continues to decrease by $\Delta$ in each period from $t+2$ provided that $\Phi\left(x^{\prime}, r v-\tau \Delta ; 1\right) \geq 0$, where $\tau$ denotes the number of periods in which the incumbents actually revise their strategies. From Lemmata $3-4, \Phi\left(x^{\prime}, r v-\tau \Delta ; 1\right) \geq$ (respectively, $<) 0$ if $x^{\prime} \leq($ respectively, $>) \chi(r v-\tau \Delta)$ and $\chi(\cdot)$ decreases with $\tau$. Therefore, there is a $\tau^{*} \in \mathbb{N}$ such that $x^{\prime} \leq \chi(r v-\tau \Delta)$ for any $\tau \in\left[1, \tau^{*}\right)$ and $x^{\prime}>\chi(r v-\tau \Delta)$ for any $\tau \in\left[\tau^{*},\left(r-\frac{N}{N-1}\right) \frac{v}{\Delta}+1\right) .{ }^{3}$ That is, every incumbent stops changing his strategy after

\footnotetext{
${ }^{3}$ The domain of the function $\chi(\cdot)$ is the interval $\left[\frac{N}{N-1} v, r v\right]$. Therefore, $r v-\tau \Delta$ must be greater than $\frac{N}{N-1} v-\Delta$, which leads to $\tau<\left(r-\frac{N}{N-1}\right) \frac{v}{\Delta}+1$.
} 
changing it in $\bar{\tau}$ periods since $\Phi\left(x^{\prime}, r v-\tau^{*} \Delta ; 1\right)<0$ if the mutant continues to choose $x^{\prime}$.

From Lemma 3 , we have $\Phi\left(x, r v-\tau^{*} \Delta ; 1\right)<0$ for any $x \in\left(\chi\left(r v-\tau^{*} \Delta\right), r v-\tau^{*} \Delta\right)$. Thus, in turn, the mutant changes into $x^{\prime}+\Delta$ at $t+\tau^{*}+1$ and continues increasing by $\Delta$ every period from $t+\tau^{*}+2$ provided that $\Phi\left(x^{\prime}+\omega \Delta, r v-\tau^{*} \Delta ; 1\right)<0$ where $\omega$ denotes the number of periods in which the mutant actually revises his strategy. In other words, the mutant increases his expenditure by $\Delta$ in every period until it reaches $r v-\tau^{*} \Delta$, or equivalently, stops changing his strategy after changing it in $\omega^{*} \equiv \frac{r v-x^{\prime}}{\Delta}-\tau^{*}$ periods. Thus, the process results in mon $\left(r v-\tau^{*} \Delta\right)$, and the transition from mon ( $\left.r v\right)$ to mon $\left(r v-\tau^{*} \Delta\right)$ can occur with one experimentation. This implies that mon $\left(r v-\tau^{*} \Delta\right)$ is an SSS.

Since $\chi(\hat{x})$ is monotone increasing, it has the inverse function of $\chi^{-1}(\bar{x})$, which is defined on the interval $[0, \chi(r v)]$. Let us define a function $\psi:[0, \chi(r v)] \cap \Gamma \longrightarrow$ $\left(\frac{N}{N-1} v-\Delta, r v\right] \cap \Gamma$ and $x^{*}=\psi(\bar{x}) \equiv \max \left\{x \in \Gamma \mid x \leq \chi^{-1}(\bar{x})\right\}$. We obtain the following lemma in regard to the function $\psi$.

Lemma 5 The function $\psi$ maps $[0, \chi(r v)] \cap \Gamma$ onto $\left(\frac{N}{N-1} v-\Delta, r v\right] \cap \Gamma$.

Proof. Suppose that there exists, contrary to our claim, $x^{\prime} \in\left(\frac{N}{N-1} v-\Delta, r v\right] \cap \Gamma$ such that $x^{\prime} \neq \psi(\bar{x})$ for all $\bar{x} \in[0, \chi(r v)] \cap \Gamma$. Note that $\psi$ is nondecreasing since $\chi^{-1}(\cdot)$ is increasing. Then, we have $x^{\prime}-\Delta=\psi(\bar{x}-\Delta)$ and $x^{\prime}+\Delta=\psi(\bar{x})$ so that $\chi^{-1}(\bar{x})$ $-\chi^{-1}(\bar{x}-\Delta)>\Delta$. Differentiating $\chi^{-1}(\bar{x})$ with respect to $\bar{x}$ yields

$$
\chi^{-1 \prime}(\bar{x})=\frac{d \hat{x}}{d \bar{x}}=-\frac{\partial \varphi / \partial \bar{x}}{\partial \varphi / \partial \hat{x}}=\frac{\bar{x}^{r-1} \hat{x}^{r} N r V-\left[\bar{x}^{r}+(N-1) \hat{x}^{r}\right]^{2}}{\bar{x}^{r} \hat{x}^{r-1} N r V-\left[\bar{x}^{r}+(N-1) \hat{x}^{r}\right]^{2}}<1,
$$

for all $\bar{x} \in[0, \chi(r v)]$ since $\bar{x}<\hat{x}$. From the mean value theorem, there is a $\bar{x}^{\prime} \in$ $(\bar{x}-\Delta, \bar{x})$ such that $\chi^{-1}(\bar{x})-\chi^{-1}(\bar{x}-\Delta)=\chi^{-1 \prime}\left(\bar{x}^{\prime}\right)[\bar{x}-(\bar{x}-\Delta)]$. Therefore, we have $\frac{\chi^{-1}(\bar{x})-\chi^{-1}(\bar{x}-\Delta)}{\Delta}=\chi^{-1 \prime}\left(\bar{x}^{\prime}\right)<1$ from (A3), or equivalently $\chi^{-1}(\bar{x})-\chi^{-1}(\bar{x}-\Delta)<\Delta$ for any $\bar{x} \in[0, \chi(r v)]$. This contradicts our assumption.

From the definition of the function $x^{*}=\psi(\bar{x})$, we can see that $x^{*}=r v-\tau^{*} \Delta$. Therefore, we obtain $\tau^{*}=\frac{r v-x^{*}}{\Delta}=\frac{r v-\psi(\bar{x})}{\Delta}$ for $\bar{x} \in[0, \chi(r v)] \cap \Gamma$. Since $\operatorname{mon}\left(r v-\tau^{*} \Delta\right)$ is the same state as mon $(\psi(\bar{x}))$, the transition from mon $(r v)$ to mon $(\psi(\bar{x}))$ can occur once a single mutant chooses any $\bar{x} \in[0, \chi(r v)] \cap \Gamma$. Lemma 5 implies that every monomorphic state in $\{\operatorname{mon}(x)\}_{x \in\left(\frac{N}{N-1} v-\Delta, r v\right] \cap \Gamma}$ is attainable without experimentation after the corre- 
sponding experimental strategy in $[0, \chi(r v)] \cap \Gamma$ is chosen by a single mutant. In other words, all monomorphic states in $\{\operatorname{mon}(x)\}_{x \in\left(\frac{N}{N-1} v-\Delta, r v\right] \cap \Gamma}$ are stochastically stable.

Next, we show that $\operatorname{mon}\left(\frac{N}{N-1} v-\Delta\right)$ is stochastically stable if $\frac{N}{N-1} v \in \Gamma$. Since $\Phi\left(0, \frac{N}{N-1} v ; 1\right)=0$, it is possible that all the $N-1$ incumbents simultaneously change their common strategy $\frac{N}{N-1} v$ into $\frac{N}{N-1} v-\Delta$ if a single player experiments the zero bid in mon $\left(\frac{N}{N-1} v\right)$. We have $\Phi\left(x, \frac{N}{N-1} v-\Delta ; 1\right)<0$ for any $x \in\left[0, \frac{N}{N-1} v-\Delta\right)$. This implies that the $N-1$ incumbents stop changing their strategies while the mutant who has chosen 0 increases his expenditure by $\Delta$ in every period until it reaches $\frac{N}{N-1} v-\Delta$.

Finally, we examine whether or not a transition from each SSS in $\{\operatorname{mon}(x)\}_{x \in\left[\frac{N}{N-1} v-\Delta, r v\right] \cap \Gamma}$ to a monomorphic state mon $(x)$ for $x>r v$ can occur with one experimentation. If such a transition can occur, then mon $(x)$ for $x>r v$ is stochastically stable. Since $\Phi(x, r v ; 1)$ $<0$ for any $x>r v$ when $r \leq \frac{N}{N-2}$, a possible transition from mon $(r v)$ to mon $(x)$ for any $x>r v$ needs more than one experimentation. Moreover, when only one mutant chooses any $x>r v$, he continues to reduce his expenditure by $\Delta$ until it reaches $r v$ while the $N-1$ incumbents continue choosing $r v$. Therefore, a state is expected to return to mon ( $r v)$ in finite periods with positive probability whenever one player decides to experiment with any $x>r v$.

Suppose that there exists a $x>r v$ such that $\Phi(x, \hat{x} ; 1) \geq 0$ for some $\hat{x} \in\left[\frac{N}{N-1} v-\Delta, r v\right)$ $\cap \Gamma$. Then, once one player experiments such an $x$, all the other players will change their expenditure to $\hat{x}+\Delta$. However, they will increase their expenditures, at most, up to $r v$ since $\Phi(x, r v ; 1)<0$ for any $x>r v$. Hence, there is no SSS in $\{\operatorname{mon}(x)\}_{x \in\left[\frac{N}{N-1} v-\Delta, r v\right) \cap \Gamma}$, which moves to mon $(x)$ for any $x>r v$ with one experimentation. Thus, we can conclude that there does not exist any SSS in the complement of $\{\operatorname{mon}(x)\}_{x \in\left[\frac{N}{N-1} v-\Delta, r v\right] \cap \Gamma}$.

Proof of Proposition 3. Clearly, no nonmonomorphic state belongs to a limit set of the unperturbed Markov process in the modified cautious imitation rule. Moreover, any monomorphic state mon $(x)$ such that $x>v$ yields a negative individual payoff, i.e., $\pi_{i}(t-1)=v-x<0$. Since $\operatorname{MIM}(t-1)=\varnothing$ when $\pi_{i}(t-1)<0$, player $i$ will target zero expenditure and decrease his expenditure by $\Delta$ in period $t$. Therefore, for every $x>v$, mon $(x)$ is not in a limit set of the unperturbed Markov process. Thus, the only candidates for an SSS are monomorphic states where all players choose identical expenditures that belong to the discrete interval $[0, v] \subset \Gamma$. Without loss of generality, the number of strategies in $[0, v]$ is assumed to be $\zeta+1$ such that $1 \leq \zeta<z$.

First, consider the case in which $0<r \leq 1$. In this case, mon $(r v)$ results in a 


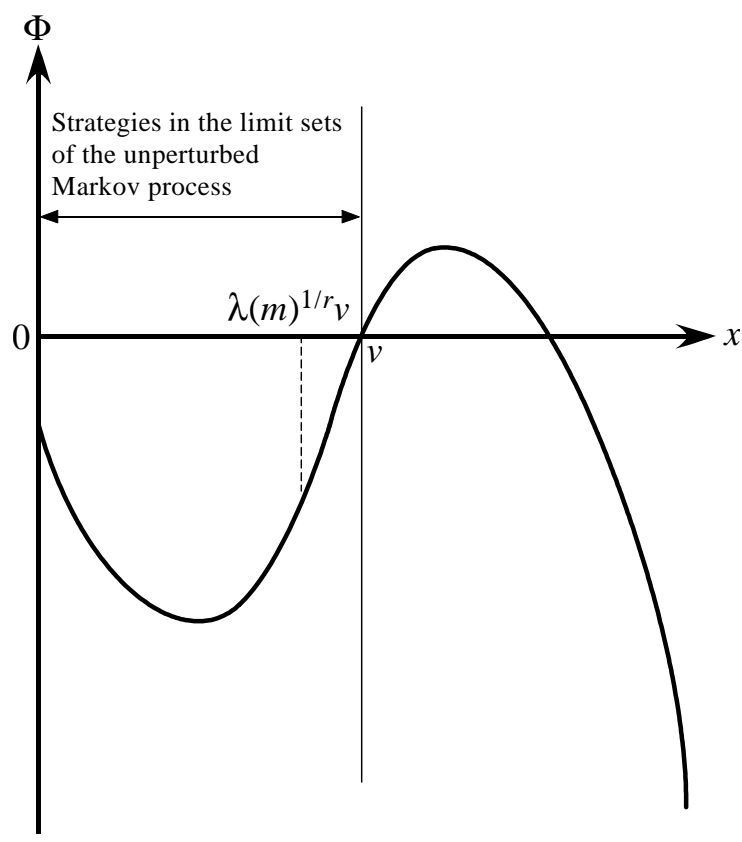

Figure 3: Configuration of the graph of the function $\Phi(x, v ; m)$ for $r>1$

nonnegative payoff. In addition, from Lemma $2, r v$ is a global maximizer of $\Phi(x, r v ; m)$ for any $r \leq 1$. Hence, like in the case of the proof of Proposition 2, we can conclude that mon $(r v)$ is a unique SSS when $r \leq 1$.

Next, consider the case in which $1<r<\infty$. From (8) and (9), we have $\Phi(0, v ; m)=$ $-m v /(N-m)<0$ and $\frac{\partial}{\partial x} \Phi(0, v ; m)=-1$, respectively. Moreover, $\Phi(x, v ; m)$ is strictly convex for $x<\lambda^{\frac{1}{r}}(m) v$ and strictly concave for $x>\lambda^{\frac{1}{r}}(m) v$. Therefore, $\Phi(x, v ; m)=0$, at most, has two real roots. Since $r>1$, we obtain $\frac{\partial}{\partial x} \Phi(v, v ; m)=r-1>0$ from (9) regardless of $m$. This implies that $x=v$ is the smaller one of the two real roots as represented in Figure 3. Hence, we obtain

$$
\Phi(x, v ; N-1)<0 \text { for any } x \in[0, v) .
$$

Thus, any transition from mon $(x)$ to mon $(v)$ for any $x \in[0, v)$ requires only one cost under the modified cautious imitation rule; this is because each of the $N-1$ incumbent players increases his expenditure by $\Delta$ until $v$ is attained. Accordingly, the cost of a tree rooted in mon $(v)$ is $\zeta$ such that mon $(v)$ is contained in a stochastically stable set of the perturbed Markov process. 
Further, we have

$$
\Phi(x, v ; 1)<0 \text { for any } x \in[0, v) .
$$

This implies that the $N-1$ incumbent players do not attempt to reduce their expenditures since one player chooses $x$ lower than $v$. On the other hand, we have $\Phi(x, v ; 1)>0$ for some $x>v$ since $\frac{\partial}{\partial x} \Phi(v, v ; 1)>0$. (See Figure 3.) Therefore, there exists an experimental strategy $x>v$ that makes each of the $N-1$ incumbent players increase his expenditure by $\Delta$. However, every player reduces his expenditure by $\Delta$ until mon $(v)$ is attained once the process reaches mon $(x)$ for any $x>v$ wherein all players obtain a negative payoff and target zero expenditure in the next period. Since all players obtain a zero payoff in mon $(v)$, the only target they can find is $v$; thus, the process returns to mon $(v)$. Hence, any transition from mon $(v)$ to mon $(x)$ for every $x \in[0, v)$ involves more than one cost; thus, mon $(x)$ for any $x \in[0, v)$ never belongs to the stochastically stable set.

\section{References}

Alós-Ferrer, C., Ania, A.B., 2005. The evolutionary stability of perfectly competitive behavior. Economic Theory 26, 497-516.

Alós-Ferrer, C., Ania, A.B., Schenk-Hoppé, K.R., 2000. An evolutionary model of Bertrand oligopoly. Games and Economic Behavior 33, 1-19.

Baye, M.R., Kovenock, D., de Vries, C.D., 1994. The solution to the Tullock rent-seeking game when $\mathrm{R}>2$ : Mixed-strategy equilibria and mean dissipation rates. Public Choice $81,363-380$.

Bergin, J., Lipman, B.L., 1996. Evolution with state-dependent mutations. Econometrica 64(4), 943-956.

Corcoran, W.J., 1984. Long-run equilibrium and total expenditures in rent-seeking. Public Choice 43, 89-94.

Corcoran, W.J., Karels, G.V., 1985. Rent-seeking behavior in the long-run. Public Choice 46, 227-246.

Foster, D., Young, P., 1990. Stochastic evolutionary game dynamics. Theoretical Population Biology 38, 219-232. 
Freidlin, M.I., Wentzell, A.D., 1984. Random perturbations of dynamic systems. SpringerVerlag, New York.

Hehenkamp, B., Leininger, W., Possajennikov, A., 2004. Evolutionary equilibrium in Tullock contests: spite and dissipation. European Journal of Political Economy 20, $1045-1057$.

Kandori, M., Mailath, G.J., Rob, R., 1993. Learning, mutation, and long run equilibria in games. Econometrica 61(1), 29-56.

Schaffer, M.E., 1988. Evolutionarily stable strategies for a finite population and a variable contest size. Journal of Theoretical Biology 132, 469-478.

Tullock, G., 1980. Efficient rent seeking. In: Buchanan, J.M., Tollison, R.D., Tullock, G. (Eds.), Toward a Theory of the Rent-Seeking Society. Texas A\&M University Press, $97-112$.

Vega-Redondo, F., 1997. The evolution of Walrasian behavior. Econometrica 65(2), 375384.

Young, P., 1993. The evolution of conventions. Econometrica 61(1), 57-84. 